\title{
Editorial
}

\section{Cancer Nanomedicine}

\author{
Haiyan Li, ${ }^{1}$ Martin M. Pike, ${ }^{2}$ Xiliang Luo, ${ }^{3}$ and Li-Hong Liu ${ }^{4}$ \\ ${ }^{1}$ Department of Physics, Portland State University, Portland, OR 97207, USA \\ ${ }^{2}$ Advanced Imaging Research Center and Department of Biomedical Engineering, Oregon Health \& Science University, \\ Portland, OR 97239, USA \\ ${ }^{3}$ Key Laboratory of Biochemical Analysis of Ministry of Education, College of Chemistry and Molecular Engineering, \\ Qingdao University of Science and Technology, Qingdao 266042, China \\ ${ }^{4}$ Department of Chemistry, Portland State University, Portland, OR 97207, USA
}

Correspondence should be addressed to Haiyan Li; haiyanl@pdx.edu and Xiliang Luo; xiliangluo@hotmail.com

Received 7 February 2013; Accepted 7 February 2013

Copyright @ 2013 Haiyan Li et al. This is an open access article distributed under the Creative Commons Attribution License, which permits unrestricted use, distribution, and reproduction in any medium, provided the original work is properly cited.

Bioengineered nanomaterials have inspired revolutionary imaging and drug delivery methods whose clinical application in cancer research has resulted in powerful medical devices for early diagnosis, treatment, and prevention of cancer. Recent advances in super imaging agents have resulted in improved resolution and sensitivity. For instance, fluorescent quantum dots with wavelength-tunable emissions, plasmon-resonant gold nanostructures with shapecontrolled near-infrared absorptions, and MRI-active iron oxide nanoparticles are well-established molecular imaging probes for noninvasive cancer imaging. Nanomaterials are also considered to be the most effective vectors that can break through transport biobarriers and deliver a constant dose of multiple therapeutic agents to tumors and intracellular endocytic compartments for cancer gene therapy, immunotherapy, or chemotherapy. Furthermore, nanowire- or nanotubebased electronic devices demonstrate extraordinary sensitivity capable of detection at the single molecule or protein level. It is anticipated that developing nanotechnology-driven imaging, sensing, and therapeutic systems will dramatically advance cancer research and clinical treatments.

We organized this special issue aiming to visualize the current progress in the emerging multidisciplinary field of cancer nanomedicine. We specifically invited Dr. R. Wang and his colleagues to analyze the trend of cancer nanomedicine development. In a review, they examined a dozen cases of particulate nanomedicine for cancer therapeutics or diagnostics applications. At least ten kinds of cancer nanomedicines have been approved by regulatory bodies to be commercialized for clinical use, and many therapeutic or theranostics cancer nanomedicines have been involved in clinical trials. These encouraging facts show that cancer nanomedicine is in action, although many technical issues need to be addressed and it is still a big challenge to meet safety guidelines for gaining clinical acceptance.

Next, we focus on some technique issues. For example, surface modification is required to stabilize and functionalize fluorescent quantum dots for cancer diagnostic and therapeutic applications. J. Wang et al. summarized some recent progress and strategies to modify quantum dots, such as silanization, ligand exchange, and amphiphilic polymer coatings. These solutions will be applied to optimize the interface chemistry of quantum dots for potential biological applications of bioimaging and drug delivery.

In a research article, J. Emami et al. demonstrated the fabrication of paclitaxel-loaded cholesterol nanostructured lipid carriers and characterization by measuring the size, zeta potential, entrapment efficiency, drug loading capacity, and drug release profiles of the carriers. The results indicate that the size of carriers is associated with oleic acid content and surfactant percent, and the ratio of drug payload to lipid weight significantly affects the entrapment efficiency and drug release from carriers.

In another research article, S. H. H. Ali et al. synthesized hippuric acid nanocomposite by intercalating hippuric acid into zinc-layered hydroxide. Compared to cytarabine alone, coupling cytarabine with hippuric acid nanocomposite has higher therapeutic efficacy against human promyelocytic 
leukemia cells. In addition, hippuric acid nanocomposite demonstrated the capability against bacteria, such as Pseudomonas aeruginosa and methicillin-resistant Staphylococcus aureus.

Recently, gold nanorods have become a rising star in cancer medicine, because under near-infrared light, the geometry-related photothermal effect enables gold nanorods to provide optical coherence tomography contrast for noninvasive cancer imaging and to ablate targeting cancer cells. In a research article, Y. Hong et al. systematically investigated how gold nanorod concentration and irradiation laser power affect the photothermal transduction efficiency of gold nanorod suspension. The therapeutic efficacy of gold nanorod-mediated ablation is estimated using MDA-MB-231 breast cancer cell line by live-dead cell staining. They also developed a versatile system that can simultaneously monitor the temperature variation, uptake of photothermal agents, and the targeting cell viability.

Toward caner immunotherapy, a gold nanoparticle arraybased sensor on chip fabricated by S. Lee and S. H. Kang can accurately detect immunomolecules by measuring the fluorescence signal from the interaction between the gold nanoparticle-captured biotin-labeled antibody and a streptavidin-labeled dye. The developed single-molecule sandwich immunoassay is simple, reliable, and sensitive (40,000-fold higher than conventional chemiluminescence immunosensors).

Interestingly, besides particulate cancer nanomedicines, M. J. Mitchell et al. demonstrated a unique device that can selectively capture and kill flowing cancer cells using microtube surfaces with immobilized halloysite nanotubes and E-selectin functionalized liposomal doxorubicin, without toxic effects on health cells. This nonparticulate cancer nanomedicine opens a new path to conduct cancer diagnosis and treatment.

The diverse and vigorous investigations published in this special issue clearly show that cancer nanomedicine is revolutionizing the field of medicine for cancers.

Haiyan Li

Martin M. Pike

Xiliang Luo

Li-Hong Liu 

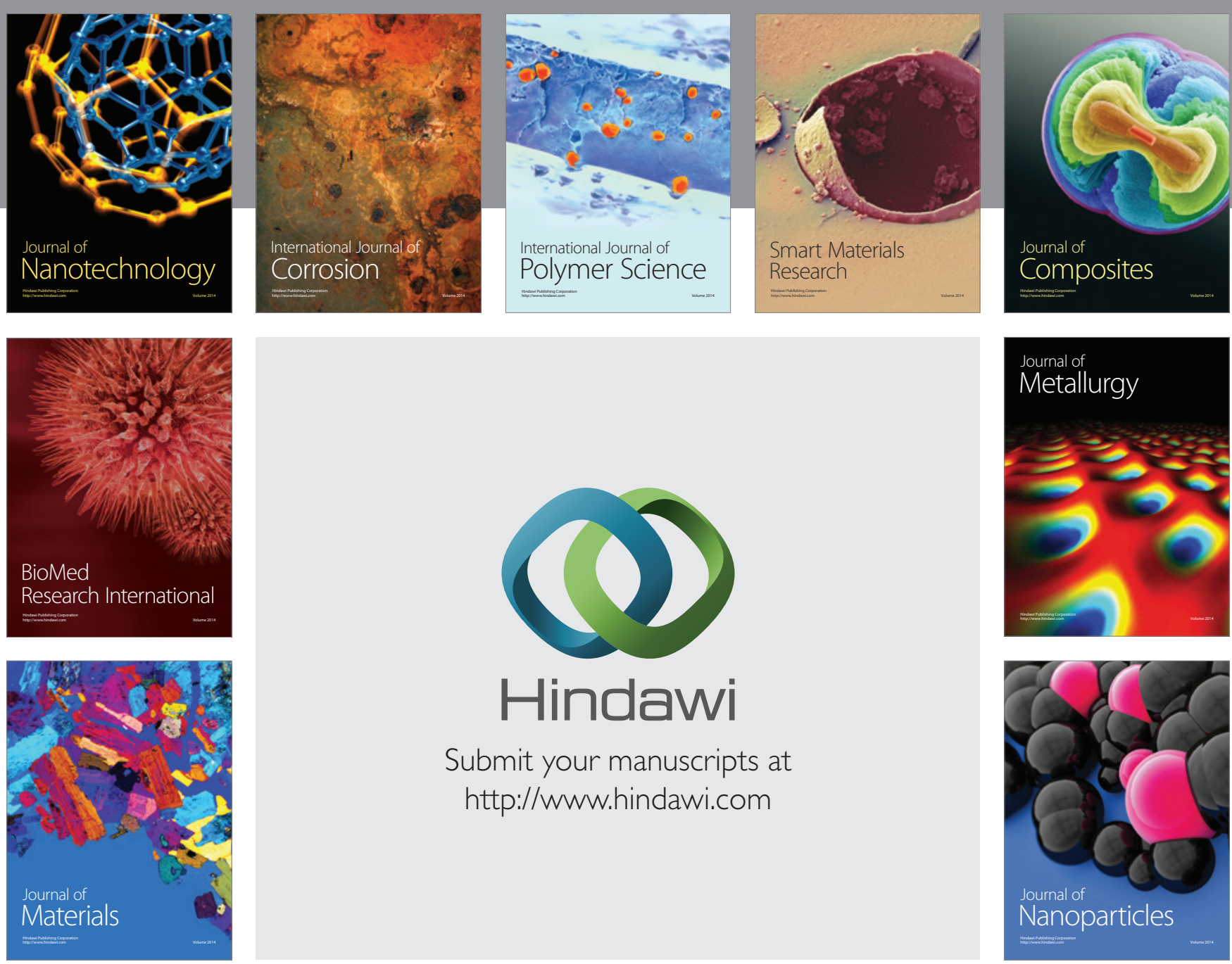

Submit your manuscripts at http://www.hindawi.com
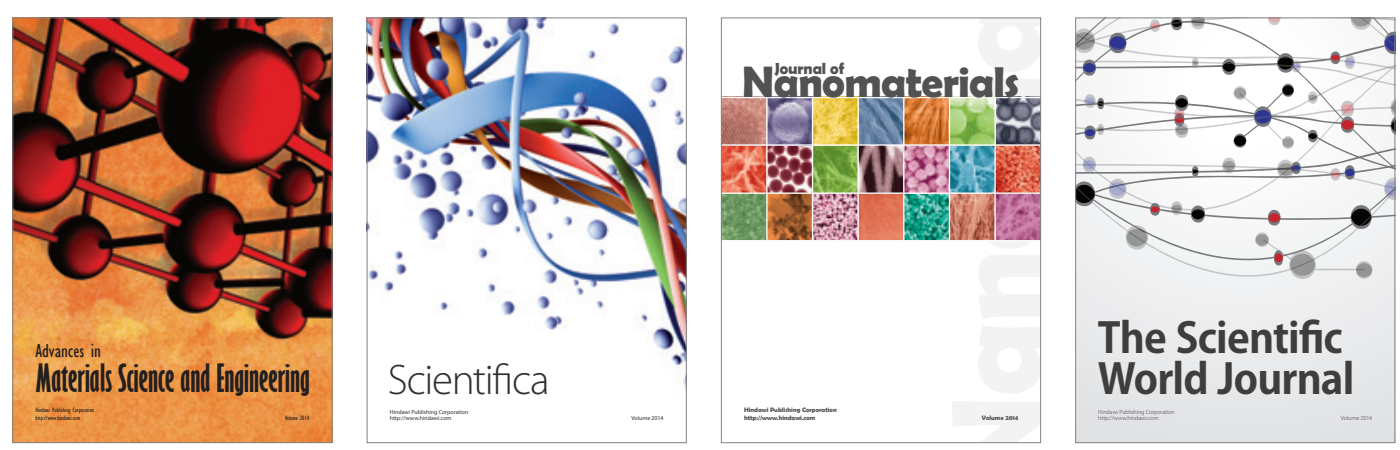

\section{The Scientific World Journal}
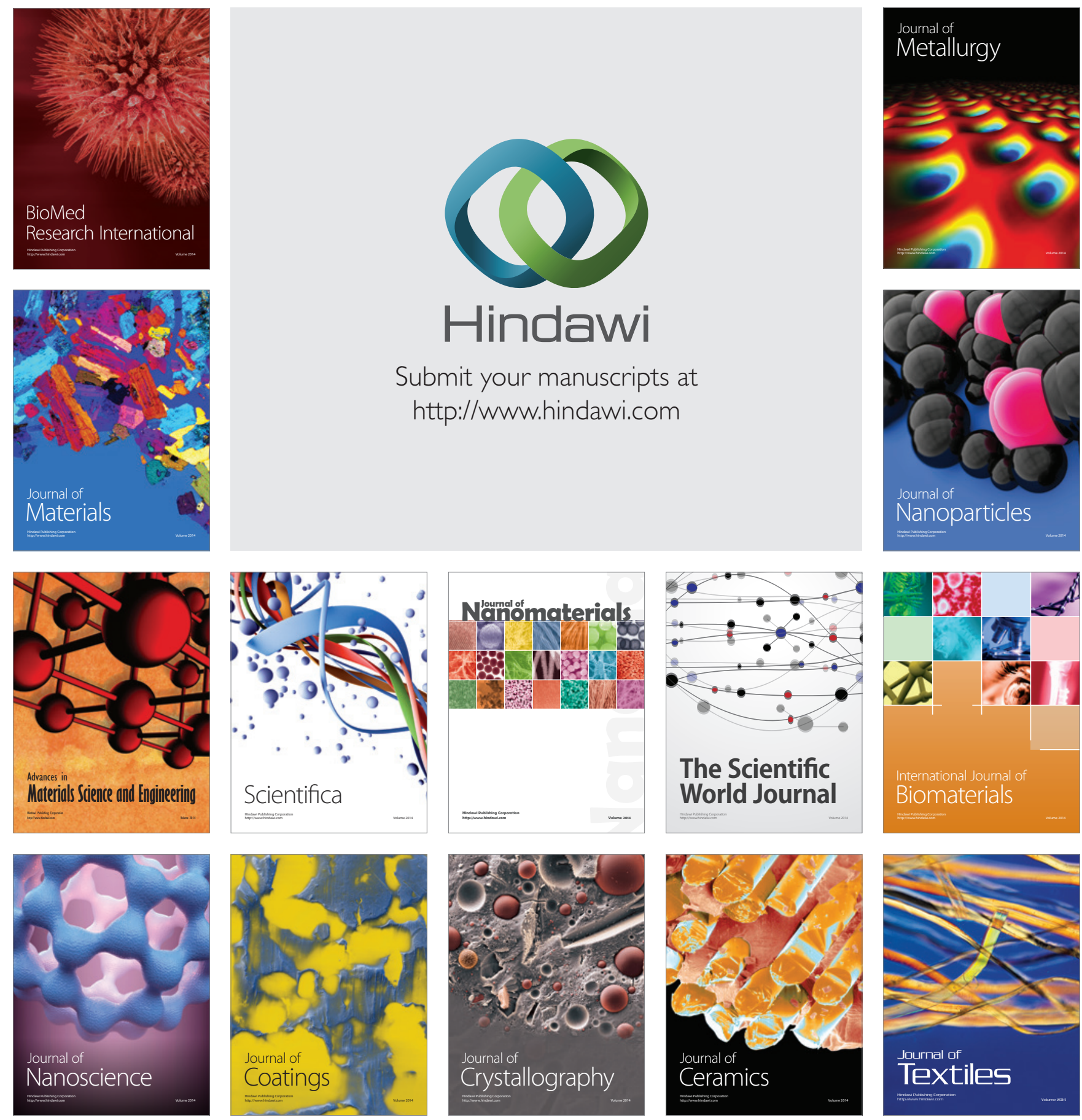